\title{
LIMIT THEOREMS FOR SUPERCRITICAL AGE-DEPENDENT BRANCHING PROCESSES WITH NEUTRAL IMMIGRATION
}

\author{
M. RICHARD, * Université Paris VI
}

\begin{abstract}
We consider a branching process with Poissonian immigration where individuals have inheritable types. At rate $\theta$, new individuals singly enter the total population and start a new population which evolves like a supercritical, homogeneous, binary CrumpMode-Jagers process: individuals have independent and identically distributed lifetime durations (nonnecessarily exponential) during which they give birth independently at a constant rate $b$. First, using spine decomposition, we relax previously known assumptions required for almost-sure convergence of the total population size. Then, we consider three models of structured populations: either all immigrants have a different type, or types are drawn in a discrete spectrum or in a continuous spectrum. In each model, the vector $\left(P_{1}, P_{2}, \ldots\right)$ of relative abundances of surviving families converges almost surely. In the first model, the limit is the GEM distribution with parameter $\theta / b$.
\end{abstract}

Keywords: Splitting tree; Crump-Mode-Jagers process; spine decomposition; immigration; structured population; GEM distribution; biogeography; almost-sure limit theorem

2010 Mathematics Subject Classification: Primary 60J80

Secondary 60G55; 92D25; 60J85; 60F15; 92D 40

\section{Introduction}

We study and give some properties about several birth-death and immigration models where immigration is structured. All individuals behave independently of one another, their lifetimes are independent and identically distributed (i.i.d.) but nonnecessarily exponential, and each female gives birth at a constant rate $b$ during her life. We will consider the supercritical case, i.e. the mean number of children of an individual is greater than 1 . In the absence of immigration, if $X(t)$ denotes the number of extant individuals at time $t$, the process $(X(t), t \geq 0)$ is a particular case of Crump-Mode-Jagers (CMJ) processes [12, Chapter 6], also called general branching processes. Here, $X$ is a binary (births arrive singly) and homogeneous (constant birth rate) CMJ process. Now, we assume that at each arrival time of a Poisson process with rate $\theta$, a new individual enters the population and starts a new population independently of the previously arrived individuals. This immigration model extends the mainland-island model of Karlin and McGregor [14] to general lifetimes. In that case, the total population process $X$ is a linear birth-and-death process with immigration. For more properties about this process, see [23] or [26] and the references therein. In the context of ecology [4], this model can be used as a null model of species diversity, in the framework of the neutral theory of biodiversity [11].

Received 2 August 2010; revision received 19 November 2010.

* Postal address: Laboratoire de Probabilités et Modèles Aléatoires, UMR 7599, Université Paris VI, Case courrier 188, 4 Place Jussieu, 75252 Paris Cedex 05, France. Email address: mathieu.richard@upmc.fr 
We first give the asymptotic behavior of the process $(I(t), t \geq 0)$ representing the total number of extant individuals on the island at time $t$. Specifically, there exists $\eta>0$ (the Malthusian parameter associated with the branching process $X)$ such that $\mathrm{e}^{-\eta t} I(t)$ converges almost surely. Tavaré [22] proved this result in the case of a linear birth process with immigration. The case of general CMJ processes was treated by Jagers [12] under the hypothesis that the variance of the number of children per individual is finite. We manage to relax this assumption in the case of homogeneous (binary) CMJ processes thanks to the spine decomposition of splitting trees [9], [16], [18], which are the genealogical trees generated by those branching processes. In passing, we obtain technical results on the log-integrability of $\sup _{t} \mathrm{e}^{-\eta t} X(t)$.

Then, we consider models where individuals bear clonally inherited types. These models intend to model a metacommunity (or mainland) which delivers immigrants to the island as in the theory of island biogeography [19]. However, we make specific assumptions about the spectrum of abundances in the metacommunity. In Model I, there is a discrete spectrum with zero macroscopic relative abundances: when an immigrant enters the population, it is of a new type each time. In Model II, we consider a discrete spectrum with nonzero macroscopic relative abundances: each type of new immigrant is chosen according to some probability ( $p_{i}$, $i \geq 1$ ). In Model III, we consider a continuous spectrum of possible types, but to enable a type to be chosen several times from the metacommunity, we change the immigration model: at each immigration time, an individual belonging to a species with abundance in $(x, x+$ $\mathrm{d} x$ ) is chosen with probability $(x f(x) / \theta) \mathrm{d} x$ (where $f$ is a positive function representing the abundance density and such that $\left.\theta:=\int_{0}^{\infty} x f(x) \mathrm{d} x<\infty\right)$, and it starts an immigration process with immigration rate $x$. The particular case of abundance density $f(x)=\mathrm{e}^{-a x} / x$ appears in many papers. Volkov et al. [24] and Watterson [25] considered $f(x)$ as a continuous equivalent of the logarithmic series distribution proposed by Fisher et al. [8] as a species abundance distribution. In this particular case, species with small abundances are often drawn, but they will have a small immigration rate.

In the three models, we obtain results for the abundances $P_{1}, P_{2}, \ldots$ of different types as time $t$ goes to $\infty$ : the vector $\left(P_{1}, P_{2}, \ldots\right)$ rescaled by the total population size converges almost surely. More precisely, in Model I, which is an extension of Tavaré's result [22] to general lifetimes, we consider the abundances of the surviving families ranked by decreasing ages and the limit follows a GEM distribution with parameter $\theta / b$. This distribution appears in other contexts: Donnelly and Tavaré [5] proved that, for a sample of size $n$ whose genealogy is described by a Kingman coalescent with mutation rate $\theta$, the frequencies of the oldest, second oldest, etc. alleles converge in distribution as $n \rightarrow \infty$ to the GEM distribution with parameter $\theta$; Ethier [7] showed that it is also the distribution of the frequencies of the alleles ranked by decreasing ages in the stationary infinitely-many-neutral-alleles diffusion model.

In a sense, the surviving families that we consider in our immigration model are 'large' families because their abundances are of the same order as the population size. Lambert [17] considered 'small' families: he gave the joint law of the number of species containing $k$ individuals, $k=1,2, \ldots$.

In Section 2 we describe the models we consider and state the results we prove in other sections. Section 3 is devoted to proving a result about the process $X$, Section 4 is devoted to proving a property of the immigration process $(I(t), t \geq 0)$, while in Section 5, we prove theorems concerning the relative abundances of types in the three models. 


\section{Preliminaries and statement of the results}

We first define splitting trees, which are random trees satisfying the following assumptions.

- Individuals behave independently from one another and have i.i.d. lifetime durations.

- Conditional on their birthdate $\alpha$ and lifespan $\zeta$, each individual reproduces according to a Poisson point process on $(\alpha, \alpha+\zeta)$ with intensity $b$.

- Births arrive singly.

We denote the common distribution of lifespan $\zeta$ by $\Lambda(\cdot) / b$, where $\Lambda$ is a positive measure on $(0, \infty)$ with mass $b$ called the lifespan measure [18].

The total population process $(X(t), t \geq 0)$ belongs to a large class of processes called CMJ processes. In these processes, also called general branching processes [12, Chapter 6], a typical individual reproduces at ages according to a random point process $\xi$ on $[0, \infty)$ (denote by $\mu:=\mathrm{E}[\xi]$ its intensity measure) and it is alive during a random time $\zeta$. Then, the CMJ process is defined as

$$
X(t)=\sum_{x} \mathbf{1}_{\left\{\alpha_{x} \leq t<\alpha_{x}+\zeta_{x}\right\}}, \quad t \geq 0,
$$

where, for any individual $x, \alpha_{x}$ is their birth time and $\zeta_{x}$ is their lifespan. In this work, the process $X$ is a homogeneous (constant birth rate) and binary CMJ process, and we obtain

$$
\mu(\mathrm{d} x)=\mathrm{d} x \int_{[x, \infty)} \Lambda(\mathrm{d} r) .
$$

We assume that the mean number of children per individual $m:=\int_{(0, \infty)} r \Lambda(\mathrm{d} r)$ is greater than 1 (supercritical case).

For $\lambda \geq 0$, define $\psi(\lambda):=\lambda-\int_{(0, \infty)}\left(1-\mathrm{e}^{-\lambda r}\right) \Lambda(\mathrm{d} r)$. The function $\psi$ is convex, differentiable on $(0, \infty), \psi\left(0^{+}\right)=0$, and $\psi^{\prime}\left(0^{+}\right)=1-\int_{0}^{\infty} r \Lambda(\mathrm{d} r)<0$. Then there exists a unique positive real number $\eta$ such that $\psi(\eta)=0$. It is seen by direct computation that this real number is a Malthusian parameter [12, p. 10], i.e. it is the finite positive solution of $\int_{0}^{\infty} \mathrm{e}^{-\eta r} \mu(\mathrm{d} r)=1$ and is such that $X(t)$ grows like $\mathrm{e}^{\eta t}$ on the survival event (see Proposition 2.1 below). From now on we define

$$
c:=\psi^{\prime}(\eta)
$$

which is positive because $\psi$ is convex.

Another branching process appears in splitting trees: if we denote by $\mathcal{Z}_{n}$ the number of individuals belonging to generation $n$ of the tree, then $\left(\mathcal{Z}_{n}, n \geq 0\right)$ is a Bienaymé-GaltonWatson process started at 1 with offspring generating function

$$
f(s):=\int_{(0, \infty)} b^{-1} \Lambda(\mathrm{d} r) \mathrm{e}^{-b r(1-s)}, \quad 0 \leq s \leq 1 .
$$

To obtain results about splitting trees and CMJ processes, Lambert [16], [18] used tree contour techniques. He proved that the contour process $Y$ of a splitting tree was a spectrally positive (i.e. with no negative jumps) Lévy process whose Laplace exponent is $\psi$. Lambert obtained results about the law of the population in a splitting tree alive at time $t$. If $\tilde{\mathrm{P}}_{x}$ denotes the law of the process $(X(t), t \geq 0)$ conditioned to start with a single ancestor living $x$ units of time,

$$
\tilde{\mathrm{P}}_{x}(X(t)=0)=\frac{W(t-x)}{W(t)},
$$


and conditional on being nonzero, $X(t)$ has a geometric distribution with success probability $1 / W(t)$, i.e. for $n \in \mathbb{N}^{*}$,

$$
\tilde{\mathrm{P}}_{x}(X(t)=n)=\left(1-\frac{W(t-x)}{W(t)}\right)\left(1-\frac{1}{W(t)}\right)^{n-1} \frac{1}{W(t)},
$$

where $W$ is the scale function [2, Chapter VII] associated with $Y$ : this is the unique, absolutely continuous, increasing function $W:[0, \infty] \rightarrow[0, \infty]$ satisfying

$$
\int_{0}^{\infty} \mathrm{e}^{-\lambda x} W(x) \mathrm{d} x=\frac{1}{\psi(\lambda)}, \quad \lambda>\eta .
$$

The two-sided exit problem can be solved thanks to this scale function:

$$
\mathrm{P}\left(T_{0}<T_{(a,+\infty)} \mid Y_{0}=x\right)=\frac{W(a-x)}{W(a)}, \quad 0<x<a,
$$

where, for a Borel set $B$ of $\mathbb{R}, T_{B}=\inf \left\{t \geq 0, Y_{t} \in B\right\}$.

We now give some properties, including the asymptotic behavior, about the CMJ process $X$.

Proposition 2.1. We denote by Ext the event $\left\{\lim _{t \rightarrow \infty} X(t)=0\right\}$.

(i) We have

$$
\mathrm{P}(\mathrm{Ext})=1-\frac{\eta}{b}
$$

and conditional on $\mathrm{Ext}^{\mathrm{c}}$,

$$
\mathrm{e}^{-\eta t} X(t) \rightarrow E \text { almost surely (a.s.) as } t \rightarrow \infty,
$$

where $E$ is an exponential random variable with parameter $c$.

(ii) If, for $x>0, \log ^{+} x:=\log x \vee 0$,

$$
\mathrm{E}\left[\left(\log ^{+} \sup _{t \geq 0}\left(\mathrm{e}^{-\eta t} X(t)\right)\right)^{2} \mid \mathrm{Ext}^{\mathrm{c}}\right]<\infty
$$

We remind the reader that $c=\psi^{\prime}(\eta)$, and then $c$ depends on the measure $\Lambda(\cdot)$. The proof of the last assertion requires involved arguments using the spine decomposition of splitting trees. Proposition 2.1, which will be proved in Section 3, is known in a particular case: if the lifetime $\Lambda(\cdot) / b$ has an exponential density with parameter $d,(X(t), t \geq 0)$ is a Markovian birth-and-death process with birth rate $b$ and death rate $d<b$. In that case, $\eta=b-d$, $c=1-d / b=\mathrm{P}\left(\mathrm{Ext}^{\mathrm{c}}\right)$, and the integrability of $\sup _{t \geq 0}\left(\mathrm{e}^{-\eta t} X(t)\right)$ stems from Doob's maximal inequality.

We now define the immigration model: let $\theta$ be a positive number, and let $0=T_{0}<T_{1}<$ $T_{2}<\cdots$ be the points of a Poisson process of rate $\theta$. At each time $T_{i}$, we assume that a new individual immigrates and starts a new population whose size evolves like $X$, independently of the other populations. That is, if, for $i \geq 1$, we call $\left(Z^{i}(t), t \geq 0\right)$ the $i$ th oldest family (the family which was started at $T_{i}$ ) then $Z^{i}(t)=X_{i}\left(t-T_{i}\right) \mathbf{1}_{\left\{t \geq T_{i}\right\}}$, where $X_{1}, X_{2}, \ldots$ are copies of $X$, and $\left(X_{i}, i \geq 1\right)$ and $\left(T_{i}, i \geq 1\right)$ are independent. See Figure 1 for a graphical representation of a splitting tree with immigration. This immigration model is a generalization of Karlin and McGregor's model [14] in the case of general lifetimes. 


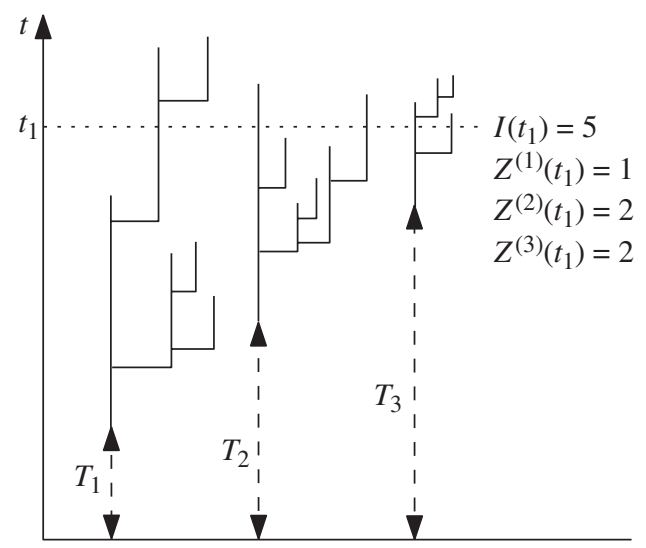

Figure 1: Splitting trees with immigration. The vertical axis is time and the horizontal axis shows filiation. At time $t_{1}$, three populations are extant.

For $i \geq 1$, denote by $\left(Z^{(i)}(t), t \geq 0\right)$ the $i$ th oldest family among the surviving populations and by $T^{(i)}$ its birthdate. In particular, by thinning of the Poisson point process, $\left(T^{(i)}, i \geq 1\right)$ is a Poisson point process with parameter $\theta \eta / b$ thanks to (2.5).

We are now interested in the joint behavior of the surviving families $\left(Z^{(i)}(t), t \geq 0\right)$ for $i \geq 1$ :

$$
\mathrm{e}^{-\eta t} Z^{(i)}(t)=\mathrm{e}^{-\eta T^{(i)}} \mathrm{e}^{-\eta\left(t-T^{(i)}\right)} Z^{(i)}(t) \stackrel{\mathrm{D}}{=} \mathrm{e}^{-\eta T^{(i)}} \mathrm{e}^{-\eta\left(t-T^{(i)}\right)} X_{(i)}\left(t-T^{(i)}\right) \mathbf{1}_{\left\{T^{(i)} \leq t\right\}} .
$$

As in (2.6), let $E_{i}:=\lim _{t \rightarrow \infty} \mathrm{e}^{-\eta t} X_{(i)}(t)$ for $i \geq 1$. Thus, $E_{1}, E_{2}, \ldots$ are i.i.d. exponential random variables with parameter $c$. Moreover, the sequences $\left(E_{i}, i \geq 1\right)$ and $\left(T^{(i)}, i \geq 1\right)$ are independent. It follows that $\mathrm{e}^{-\eta t} Z^{(i)}(t) \rightarrow \mathrm{e}^{-\eta T^{(i)}} E_{i}$ a.s. as $t \rightarrow \infty$. We record this in the following proposition.

Proposition 2.2. We have

$$
\mathrm{e}^{-\eta t}\left(Z^{(1)}(t), Z^{(2)}(t), \ldots\right) \rightarrow\left(\mathrm{e}^{-\eta T^{(1)}} E_{1}, \mathrm{e}^{-\eta T^{(2)}} E_{2}, \ldots\right) \text { a.s. as } t \rightarrow \infty,
$$

where the $E_{i} s$ are independent copies of $E$ and independent of the $T^{(i)} s$.

For $t \geq 0$, let $I(t)$ be the size of the total population at time $t$, i.e.

$$
I(t)=\sum_{i \geq 1} Z^{i}(t)
$$

The process $(I(t), t \geq 0)$ is a non-Markovian continuous-time branching process with immigration.

Theorem 2.1. (i) For $t$ positive, I ( $t$ ) has a negative binomial distribution with parameters $1-W(t)^{-1}$ and $\theta / b$, i.e. for $s \in[0,1]$, its generating function is

$$
G_{t}(s):=\mathrm{E}\left[s^{I(t)}\right]=\left(\frac{W(t)^{-1}}{1-s\left(1-W(t)^{-1}\right)}\right)^{\theta / b} .
$$


(ii) We have

$$
I:=\lim _{t \rightarrow \infty} \mathrm{e}^{-\eta t} I(t)=\sum_{i \geq 1} \mathrm{e}^{-\eta T^{(i)}} E_{i} \quad \text { a.s. },
$$

and I has a gamma distribution $\Gamma(\theta / b, c)$, i.e. the density of I with respect to the Lebesgue measure is

$$
g(x)=\frac{c^{\theta / b} x^{\theta / b-1} \mathrm{e}^{-c x}}{\Gamma(\theta / b)}, \quad x>0 .
$$

Theorem 2.1(i) is a generalization of a result by Kendall [15] which was the particular Markovian case of a birth, death and immigration process. The proof we give in Section 4 uses (2.1) and (2.2) about the law of $X(t)$.

There exist other proofs of the almost-sure convergence in Theorem 2.1(ii), but they require stronger assumptions. For example, Jagers [12, p. 198] gave a proof for the convergence of general branching processes with immigration under the hypothesis that the variance of the number of children per individual $\xi(\infty)$ is finite. In our case, this is only true if $\int_{(0, \infty)} r^{2} \Lambda(\mathrm{d} r)<\infty$. In the particular Markovian case described previously, the proof is also easier since $\left(\mathrm{e}^{-\eta t} X(t)\right.$, $t \geq 0)$ is a nonnegative martingale [1, p. 111], $\left(\mathrm{e}^{-\eta t} I(t), t \geq 0\right)$ is a nonnegative submartingale, and both converge a.s. In the proof we give in Section 4, the only assumption we use about the measure $\Lambda$ is that its mass is finite. The proof is based on Proposition 2.1(ii).

In the following, we will consider different kinds of metacommunity where immigrants are chosen and we will give results about abundances of surviving populations. In Model I, there is a discrete spectrum with zero macroscopic relative abundances: when a new family is initiated, it is of a type different from that of any previous family. The following theorem yields the asymptotic behaviors of the fractions of the surviving subpopulations ranked by decreasing ages in the total population.

Theorem 2.2. (Model I.) We have

$$
\lim _{t \rightarrow \infty} I(t)^{-1}\left(Z^{(1)}(t), Z^{(2)}(t), \ldots\right)=\left(P_{1}, P_{2}, \ldots\right) \text { a.s. }
$$

where the law of $\left(P_{1}, P_{2}, \ldots\right)$ is a GEM distribution with parameter $\theta / b$. In other words, for $i \geq 1$,

$$
P_{i} \stackrel{\mathrm{D}}{=} B_{i} \prod_{j=1}^{i-1}\left(1-B_{j}\right)
$$

and $\left(B_{i}\right)_{i \geq 1}$ is a sequence of i.i.d. random variables with law $\operatorname{Beta}(1, \theta / b)$ whose density with respect to the Lebesgue measure is

$$
\frac{\theta}{b}(1-x)^{\theta / b-1} \mathbf{1}_{[0,1]}(x) .
$$

This result was proved by Tavaré [22] in the case where $\Lambda(\mathrm{d} r)=\delta_{\infty}(\mathrm{d} r)$ (pure-birth process); it is the exponential case defined previously with $b=1$ and $d=0$. His result is robust because we see that in our more general case, the limit distribution does not depend on the lifespan distribution but only on the immigration-to-birth ratio $\theta / b$. In biogeography, a typical problem is to recover data about population dynamics (immigration times, law of lifespan duration) from the observed diversity patterns. In this model, we see that there is a loss of information about the lifespan duration. However, the ratio $\theta / b$ can be estimated thanks to the species abundance distribution. We will prove Theorem 2.2 in Subsection 5.1. 
In Model II, we consider a discrete spectrum with nonzero macroscopic relative abundances. Contrary to Model I where types were always new, they are now given a priori and types of immigrants are independently drawn according to some probability $p=\left(p_{i}, i \geq 1\right)$. When a population is initiated (i.e. at each time of the $\theta$-Poisson point process), it is of type $i$ with probability $p_{i}>0$.

Theorem 2.3. (Model II.) For $i \geq 1$, denote by $I_{i}(t)$ the number of individuals of type $i$ at time $t$ and $\operatorname{set} \theta_{i}:=\theta p_{i} / b$. Then

$$
\lim _{t \rightarrow \infty} I(t)^{-1}\left(I_{1}(t), I_{2}(t), \ldots\right)=\left(P_{1}^{\prime}, P_{2}^{\prime}, \ldots\right) \text { a.s. }
$$

where, for $i \geq 1$,

$$
P_{i}^{\prime} \stackrel{\mathrm{D}}{=} B_{i}^{\prime} \prod_{j=1}^{i-1}\left(1-B_{j}^{\prime}\right)
$$

and $\left(B_{i}^{\prime}\right)_{i \geq 1}$ is a sequence of independent random variables such that

$$
B_{i}^{\prime} \sim \operatorname{Beta}\left(\theta_{i}, \frac{\theta}{b} \sum_{j \geq i+1} p_{j}\right)
$$

In particular, for $i \geq 1, P_{i}^{\prime}$ has a beta distribution $\operatorname{Beta}\left(\theta_{i}, \theta / b-\theta_{i}\right)$.

The proof of this theorem will be given in Subsection 5.2. In this model, the limit depends only on $\theta / b$ and the metacommunity spectrum $\left(p_{i}, i \geq 1\right)$.

Remark 2.1. If the number of possible types $n$ is finite then

$$
\sum_{i=1}^{n} P_{i}^{\prime}=\sum_{i=1}^{n} \frac{I_{i}}{I}=1, \quad \sum_{i=1}^{n} \theta_{i}=\frac{\theta}{b}
$$

and the joint density of $\left(P_{1}^{\prime}, \ldots, P_{n}^{\prime}\right)$ is

$$
\frac{\Gamma(\theta / b)}{\prod_{i=1}^{n} \Gamma\left(\theta_{i}\right)}\left(\prod_{i=1}^{n-1} x_{i}^{\theta_{i}-1} \mathbf{1}_{\left\{x_{i}>0\right\}}\right)\left(1-x_{1}-\cdots-x_{n-1}\right)^{\theta_{n}-1} \mathbf{1}_{\left\{x_{1}+\cdots+x_{n-1}<1\right\}} .
$$

This is the joint density of a Dirichlet distribution $\operatorname{Dir}\left(\theta_{1}, \ldots, \theta_{n}\right)$.

In Model III, we consider a continuous spectrum of possible types and we slightly modify the immigration process: when an individual arrives on the island, it starts a new population with an immigration rate proportional to its abundance on the metacommunity. More precisely, let $\Pi$ be a Poisson point process on $\mathbb{R}_{+} \times \mathbb{R}_{+}$with intensity $\mathrm{d} t \otimes x f(x) \mathrm{d} x$, where $f$ is a nonnegative function such that $\theta:=\int_{0}^{\infty} x f(x) \mathrm{d} x$ is finite. Then, write $\Pi:=\left(\left(T_{i}, \Delta_{i}\right), i \geq 1\right)$, where $T_{1}<T_{2}<\cdots$ are the times of a $\theta$-linear Poisson point process and $\left(\Delta_{i}, i \geq 1\right)$ is a sequence of i.i.d. random variables whose density is $\theta^{-1} x f(x) \mathrm{d} x$, which is independent of $\left(T_{i}, i \geq 1\right)$. At time $T_{i}$, a new population starts out and it evolves like the continuous-time branching process with immigration defined for the two previous models with an immigration rate $\Delta_{i}$. The interpretation of this model is as follows: for $x>0, f(x)$ represents the density of species with abundance $x$ in the metacommunity and at each immigration time, an individual of 
a species with abundance in $(x, x+\mathrm{d} x)$ is chosen with probability $(x f(x) / \theta) \mathrm{d} x$ proportional to its abundance in the metacommunity.

If $\left(Z^{i}(t), t \geq 0\right)$ is the $i$ th oldest family,

$$
Z^{i}(t)=I_{\Delta_{i}}^{i}\left(t-T_{i}\right) \mathbf{1}_{\left\{t \geq T_{i}\right\}}, \quad t \geq 0,
$$

where the $I_{\Delta_{i}}^{i}$ s are independent copies of $I_{\Delta}$, which, conditional on $\Delta$, evolves like the immigration process of the first two models with an immigration rate $\Delta$. According to Theorem 2.1(ii), we know that

$$
\mathrm{e}^{-\eta t} I_{\Delta}(t) \rightarrow G \quad \text { a.s. as } t \rightarrow \infty,
$$

where, conditional on $\Delta, G \sim \operatorname{Gamma}(\Delta / b, c)$. We denote by $F$ its distribution tail

$$
F(v):=\mathrm{P}(G \geq v)=\int_{0}^{\infty} \mathrm{d} x \frac{x f(x)}{\theta} \int_{v}^{\infty} \frac{\mathrm{e}^{-c t} t^{x / b-1} c^{x / b}}{\Gamma(x / b)} \mathrm{d} t .
$$

Hence, we also have the following result.

Proposition 2.3. For $i \geq 1$,

$$
\mathrm{e}^{-\eta t} Z^{i}(t) \rightarrow \mathrm{e}^{-\eta T_{i}} G_{i} \quad \text { a.s. as } t \rightarrow \infty
$$

where $\left(G_{i}, i \geq 1\right)$ is a sequence of i.i.d. random variables with the same distribution as $G$ and independent of $\left(T_{i}, i \geq 1\right)$.

We again denote by $I(t)$ the total population at time $t$, i.e. $I(t):=\sum_{i \geq 1} Z^{i}(t)$, and we obtain a result similar to Theorem 2.1 concerning the asymptotic behavior of $I(t)$.

Proposition 2.4. If $\int_{0}^{\infty} x^{2} f(x) \mathrm{d} x<\infty$, we have

$$
\mathrm{e}^{-\eta t} I(t) \rightarrow \sum_{i \geq 1} \mathrm{e}^{-\eta T_{i}} G_{i} \quad \text { a.s. as } t \rightarrow \infty,
$$

and the Laplace transform of $\sigma:=\sum_{i \geq 1} \mathrm{e}^{-\eta T_{i}} G_{i}$ is

$$
\mathrm{E}\left[\mathrm{e}^{-s \sigma}\right]=\exp \left(-\frac{\theta}{\eta} \int_{0}^{\infty} \frac{F(v)}{v}\left(1-\mathrm{e}^{-s v}\right) \mathrm{d} v\right) .
$$

Moreover,

$$
\mathrm{E}[\sigma]=\frac{1}{\eta b c} \int_{0}^{\infty} x^{2} f(x) \mathrm{d} x<\infty .
$$

We also have a result about abundances of different types.

Theorem 2.4. (Model III.) We have

$$
\left(\frac{Z^{1}(t)}{I(t)}, \frac{Z^{2}(t)}{I(t)}, \ldots\right) \rightarrow\left(\frac{\sigma_{1}}{\sigma}, \frac{\sigma_{2}}{\sigma}, \ldots\right) \text { a.s. as } t \rightarrow \infty
$$

where $\left(\sigma_{i}, i \geq 1\right)$ are the points of a nonhomogeneous Poisson point process on $(0, \infty)$ with intensity measure $(\theta / \eta F(y) / y) \mathrm{d} y$ and $\sigma=\sum_{i \geq 1} \sigma_{i}$.

The proofs of these two results will be given in Subsection 5.3. Note that in this model, the limit depends only on the lifespan measure via the Malthusian parameter $\eta$ and the constant $c$. 


\section{Proof of Proposition 2.1}

\subsection{Some useful, technical lemmas}

We now state some lemmas that will be useful in subsequent proofs.

Lemma 3.1. Let $Y_{1}, Y_{2}, \ldots$ be a sequence of i.i.d. random variables with finite expectation. Then, if $S:=\sup _{n \geq 1}\left((1 / n) \sum_{i=1}^{n} Y_{i}\right)$,

$$
\mathrm{E}\left[\left(\log ^{+} S\right)^{k}\right]<\infty, \quad k \geq 1 .
$$

Proof. According to Kallenberg [13, p. 184], for $r>0$,

$$
r \mathrm{P}(S \geq 2 r) \leq \mathrm{E}\left[Y_{1} ; Y_{1} \geq r\right] .
$$

Hence, choosing $r=\mathrm{e}^{s} / 2$, we have, for $s \geq 0$,

$$
\mathrm{P}\left(\log ^{+} S \geq s\right) \leq 2 \mathrm{e}^{-s} \mathrm{E}\left[Y_{1}\right]
$$

and

$$
\mathrm{E}\left[\left(\log ^{+} S\right)^{k}\right]=\int_{0}^{\infty} k s^{k-1} \mathrm{P}\left(\log ^{+} S \geq s\right) \mathrm{d} s \leq 2 \mathrm{E}\left[Y_{1}\right] k \int_{0}^{\infty} s^{k-1} \mathrm{e}^{-s} \mathrm{~d} s<\infty .
$$

This completes the proof.

Lemma 3.2. Let $A$ be a homogeneous Poisson process with parameter $\rho$. If $S:=\sup _{t>0}\left(A_{t} / t\right)$ then, for $a>0$,

$$
\mathrm{P}(S>a)=\frac{\rho}{a} \vee 1
$$

In particular,

$$
\mathrm{E}\left[\left(\log ^{+} S\right)^{k}\right]<\infty \text { for all } k \geq 1 .
$$

Proof. If $a<\rho$, since $\lim _{t \rightarrow \infty} A_{t} / t=\rho, \mathrm{P}(S>a)=1$. Now let $a$ be a real number greater than $\rho$. Then

$$
\mathrm{P}(S \leq a)=\mathrm{P}\left(\text { for all } t \geq 0, \text { at }-A_{t} \geq 0\right) .
$$

According to Bertoin [2, Chapter VII], since ( $\left.a t-A_{t}, t>0\right)$ is a Lévy process with no positive jumps and with Laplace exponent $\phi(\lambda)=\lambda a-\rho\left(1-\mathrm{e}^{-\lambda}\right)$, we have, as in (2.4),

$$
\mathrm{P}(S \leq a)=\frac{H(0)}{H(\infty)}
$$

where $H$ is the scale function associated with $\left(a t-A_{t}, t>0\right)$. We compute $H(0)$ and $H(\infty)$ using Tauberian theorems [2, p. 10]:

- since $\phi(\lambda) \sim \lambda(a-\rho)$ as $\lambda \rightarrow 0$, then $H(\infty)=(a-\rho)^{-1}$,

- since $\phi(\lambda) \sim \lambda a$ as $\lambda \rightarrow 0$, then $H(0)=a^{-1}$.

Hence,

$$
\mathrm{P}(S \leq a)=\frac{a-\rho}{a}=1-\frac{\rho}{a}, \quad a>\rho .
$$


Then,

$$
\begin{aligned}
\mathrm{E}\left[\left(\log ^{+} S\right)^{k}\right] & =\int_{0}^{\infty} k r^{k-1} \mathrm{P}\left(\log ^{+} S \geq r\right) \mathrm{d} r \\
& \leq \int_{0}^{\log ^{+} \rho} k r^{k-1} \mathrm{~d} r+\int_{\log ^{+} \rho}^{\infty} k r^{k-1} \frac{\rho}{\mathrm{e}^{r}} \mathrm{~d} r \\
& <\infty
\end{aligned}
$$

and the proof is completed.

\subsection{Proof of Proposition 2.1(i)}

Lambert proved in [16] that $\mathrm{P}(\mathrm{Ext})=1-\eta / b$ and in [18] that, conditional on $\mathrm{Ext}^{\mathrm{c}}$,

$$
\mathrm{e}^{-\eta t} X(t) \stackrel{\mathrm{D}}{\rightarrow} E \quad \text { as } t \rightarrow \infty
$$

where $E$ is an exponential random variable with parameter $c$. To obtain almost-sure convergence, we use [20, Theorem 5.4], in which Nerman gave sufficient conditions for convergence of CMJ processes to hold a.s. Here, the two conditions of his theorem are satisfied. Indeed, the second condition holds if there exists on $[0, \infty)$ an integrable, bounded, nonincreasing positive function $h$ such that

$$
\mathrm{E}\left[\sup _{t \geq 0}\left(\frac{\mathrm{e}^{-\eta t} \mathbf{1}_{\{t<\zeta\}}}{h(t)}\right)\right]<\infty,
$$

where we recall that $\zeta$ is the lifespan duration of a typical individual in the CMJ process $X$. Then, choosing $h(t)=\mathrm{e}^{-\eta t}$, this condition is trivially satisfied.

The first condition holds if there exists a nonincreasing Lebesgue integrable positive function $g$ such that

$$
\int_{0}^{\infty} \frac{1}{g(t)} \mathrm{e}^{-\eta t} \mu(\mathrm{d} t)<\infty .
$$

Taking $g(t)=\mathrm{e}^{-\beta t}$ with $\eta>\beta>0$ and recalling that $\mu(\mathrm{d} t)=\int_{(t, \infty)} \Lambda(\mathrm{d} r) \mathrm{d} t$, we have

$$
\begin{aligned}
\int_{0}^{\infty} \frac{1}{g(t)} \mathrm{e}^{-\eta t} \mu(\mathrm{d} t) & =\int_{0}^{\infty} \mathrm{e}^{(\beta-\eta) t} \int_{t}^{\infty} \Lambda(\mathrm{d} r) \mathrm{d} t \\
& =\int_{(0, \infty)} \Lambda(\mathrm{d} r) \int_{0}^{r} \mathrm{e}^{(\beta-\eta) t} \mathrm{~d} t \\
& \leq C \int_{(0, \infty)} \Lambda(\mathrm{d} r) \\
& =C b \\
& <\infty
\end{aligned}
$$

and condition (3.1) is fulfilled.

\subsection{Proof of Proposition 2.1(ii)}

We will say that a process $G$ satisfies condition (C) if

$$
\mathrm{E}\left[\left(\log ^{+} \sup _{t \geq 0} \mathrm{e}^{-\eta t} G_{t}\right)^{2}\right]<\infty,
$$


and our aim is to prove that, conditional on nonextinction, the homogeneous CMJ process $(X(t), t \geq 0)$ satisfies it.

According to Theorem 4.4.1.1 of [16], conditional on the nonextinction of $(X(t), t \geq 0)$,

$$
X(t)=X_{t}^{\infty}+X_{t}^{d}+X_{t}^{g},
$$

where we have used the following notation.

- $X_{t}^{\infty}$ is the number of individuals alive at time $t$ and whose descendance is infinite. In particular, $\left(X_{t}^{\infty}, t \geq 0\right)$ is a Yule process with rate $\eta$.

- $X_{t}^{d}$ is the number of individuals alive at time $t$ descending from trees grafted on the right-hand side of the Yule tree (here right refers to the order of the contour of the planar splitting tree)

$$
X_{t}^{d}:=\sum_{i=1}^{\tilde{N}_{t}} \tilde{X}_{i}\left(t-\tilde{T}_{i}\right)
$$

where

- $\left(\tilde{X}_{i}, i \geq 1\right)$ is a sequence of i.i.d. splitting trees conditional on extinction and independent of $X^{\infty}$; we know that such trees have the same distribution as subcritical splitting trees with lifespan measure $\tilde{\Lambda}(\mathrm{d} r)=\mathrm{e}^{-\eta r} \Lambda(\mathrm{d} r)$ (cf. [16]),

- conditionally on $\left(X_{t}^{\infty}, t \geq 0\right),\left(\tilde{N}_{t}, t \geq 0\right)$ is a nonhomogeneous Poisson process with mean measure $(b-\eta) X_{t}^{\infty} \mathrm{d} t$ and independent of $\left(\tilde{X}_{i}\right)_{i}$; we denote its arrival times by $\tilde{T}_{1}, \tilde{T}_{2}, \ldots$

- $X_{t}^{g}$ is the number of individuals alive at time $t$ descending from trees grafted on the lefthand side of the Yule tree (here left also refers to the contour order). More specifically, let $(A, R)$ be a couple of random variables with joint law given by

$$
\mathrm{P}(A+R \in \mathrm{d} z, R \in \mathrm{d} r)=\mathrm{e}^{-\eta r} \mathrm{~d} r \Lambda(\mathrm{d} z), \quad 0<r<z,
$$

and let $\left(\left(A_{i, j}, R_{i, j}\right), i \geq 0, j \geq 1\right)$ be i.i.d. random variables distributed as $(A, R)$. We consider the arrival times

$$
T_{i, j}=\tau_{i}+A_{i, 1}+A_{i, 2}+\cdots+A_{i, j}, \quad i \geq 0, j \geq 1,
$$

where $0=\tau_{0}<\tau_{1}<\tau_{2}<\cdots$ are the splitting times of the Yule tree, that is, on each new infinite branch, we start a new $A$-renewal process independent of the others. We define, for $t \geq 0$,

$$
X_{t}^{g}:=\sum_{i, j} \hat{X}_{i, j}\left(t-T_{i, j}\right) \mathbf{1}_{\left\{T_{i, j} \geq t\right\}},
$$

where $\left(\hat{X}_{i, j}, i \geq 0, j \geq 1\right)$ is a sequence of i.i.d. splitting trees independent of $X^{\infty}$, conditioned on extinction and such that the unique ancestor of $\hat{X}_{i, j}$ has lifetime $R_{i, j}$. We denote by $\hat{N}_{t}:=\#\left\{(i, j), T_{i, j} \leq t\right\}$ the number of graft times before $t$.

This spine decomposition is represented in Figure 2.

In the following, we will prove that $\left(X_{t}, t \geq 0\right)$ satisfies condition (C). To do this, using Minkowski inequality and the inequality

$$
\log ^{+}(x+y) \leq \log ^{+} x+\log ^{+} y+\log 2 \text { for all } x, y \geq 0,
$$

we only have to check that the three processes $X^{\infty}, X^{g}$, and $X^{d}$ satisfy condition (C). 


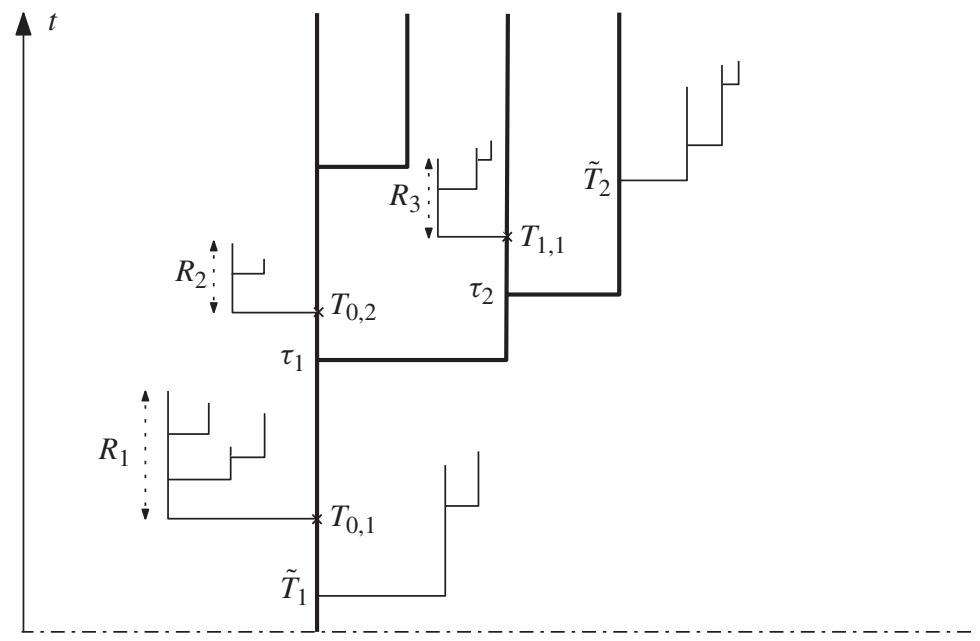

FIGURE 2: Spine decomposition of a splitting tree. The thick lines represent the Yule tree $X^{\infty}$ on which we graft on the left (at times $T_{0,1}, \ldots$ ) the trees conditioned on extinction whose ancestors have lifetime durations distributed as $R$ and on the right (at time $\tilde{T}_{1}, \ldots$ ) the trees conditioned on extinction.

3.3.1. Proof of condition $(C)$ for $X^{\infty}$. Since $\left(X_{t}^{\infty}, t \geq 0\right)$ is an $\eta$-Yule process, $\left(\mathrm{e}^{-\eta t} X_{t}^{\infty}\right.$, $t \geq 0)$ is a nonnegative martingale [1, p. 111] and so, by Doob's inequality [21, p. 54],

$$
\mathrm{E}\left[\sup _{t \geq 0}\left(\mathrm{e}^{-\eta t} X_{t}^{\infty}\right)^{2}\right] \leq 4 \sup _{t \geq 0} \mathrm{E}\left[\left(\mathrm{e}^{-\eta t} X_{t}^{\infty}\right)^{2}\right] .
$$

Moreover, $\mathrm{E}\left[\left(X_{t}^{\infty}\right)^{2}\right]=2\left(\mathrm{e}^{2 \eta t}-\mathrm{e}^{\eta t}\right)$ (again in [1]) and

$$
\mathrm{E}\left[\left(\mathrm{e}^{-\eta t} X_{t}^{\infty}\right)^{2}\right]=2 \mathrm{e}^{-2 \eta t}\left(\mathrm{e}^{2 \eta t}-\mathrm{e}^{\eta t}\right) \rightarrow 2 \text { as } t \rightarrow \infty .
$$

Hence, the supremum on the right-hand side of (3.3) is finite and

$$
\mathrm{E}\left[\left(\sup _{t \geq 0}\left(\mathrm{e}^{-\eta t} X_{t}^{\infty}\right)\right)^{2}\right]<\infty .
$$

From now on, we will set $M:=\sup _{t \geq 0} \mathrm{e}^{-\eta t} X_{t}^{\infty}$. Since $\mathrm{E}\left[M^{2}\right]<\infty$, condition (C) is trivially satisfied by $X^{\infty}$.

3.3.2. Proof of condition $(C)$ for $X^{d}$. We recall that

$$
X_{t}^{d}=\sum_{i=1}^{\tilde{N}_{t}} \tilde{X}_{i}\left(t-\tilde{T}_{i}\right)
$$

Denote by $Y_{i}$ the total progeny of the conditioned splitting tree $\tilde{X}_{i}$, that is, the total number of descendants of the ancestor plus 1 . Then, a.s., for all $t \geq 0$ and $i \geq 1$, we have $\tilde{X}_{i}\left(t-\tilde{T}_{i}\right) \leq Y_{i}$ and

$$
X_{t}^{d} \leq \sum_{i=1}^{\tilde{N}_{t}} Y_{i} \quad \text { a.s. for } t \geq 0 .
$$


Hence, a.s. for all $t$,

$$
\mathrm{e}^{-\eta t} X_{t}^{d} \leq \mathrm{e}^{-\eta t} \sum_{i=1}^{\tilde{N}_{t}} Y_{i}=\mathrm{e}^{-\eta t} \tilde{N}_{t}\left(\frac{1}{\tilde{N}_{t}} \sum_{i=1}^{\tilde{N}_{t}} Y_{i}\right)
$$

and, thanks to Minkowski's inequality,

$$
\begin{aligned}
\mathrm{E}\left[\left(\log ^{+} \sup _{t \geq 0}\left(\mathrm{e}^{-\eta t} X_{t}^{d}\right)\right)^{2}\right]^{1 / 2} \leq & \mathrm{E}\left[\left(\log ^{+} \sup _{t \geq 0}\left(\mathrm{e}^{-\eta t} \tilde{N}_{t}\right)\right)^{2}\right]^{1 / 2} \\
& +\mathrm{E}\left[\left(\log ^{+} \sup _{t>0}\left(\frac{1}{\tilde{N}_{t}} \sum_{i=1}^{\tilde{N}_{t}} Y_{i}\right)\right)^{2}\right]^{1 / 2} .
\end{aligned}
$$

We first consider the second term on the right-hand side of (3.4). We have

$$
\mathrm{E}\left[\left(\log ^{+} \sup _{t>0}\left(\frac{1}{\tilde{N}_{t}} \sum_{i=1}^{\tilde{N}_{t}} Y_{i}\right)\right)^{2}\right] \leq \mathrm{E}\left[\left(\log ^{+} \sup _{n \geq 1}\left(\frac{1}{n} \sum_{i=1}^{n} Y_{i}\right)\right)^{2}\right]
$$

since $\left(\tilde{N}_{t}, t \geq 0\right)$ is integer valued. Thanks to Lemma 3.1, this term is finite because $\mathrm{E}\left[Y_{1}\right]$ is finite. Indeed, $Y_{1}$ is the total progeny of a subcritical branching process and it is known [10, p. 32] that its mean is finite.

We are now interested in the first term on the right-hand side of (3.4). We work conditionally on $X^{\infty}=:(f(t), t \geq 0)$. Since we have $\mathrm{e}^{-\eta t} \int_{0}^{t} f(s) \mathrm{d} s \leq M$ using the supremum $M$ of $\left(\mathrm{e}^{-\eta t} X_{t}^{\infty}, t \geq 0\right)$

$$
\mathrm{e}^{-\eta t} \tilde{N}_{t}=\mathrm{e}^{-\eta t} \int_{0}^{t} f(s) \mathrm{d} s \frac{\tilde{N}_{t}}{\int_{0}^{t} f(s) \mathrm{d} s} \leq M \frac{\tilde{N}_{t}}{\int_{0}^{t} f(s) \mathrm{d} s} .
$$

Moreover,

$$
\left(\tilde{N}_{t}, t \geq 0\right) \stackrel{\mathrm{D}}{=}\left(N_{\int_{0}^{t} f(s) \mathrm{d} s}^{\prime}, t \geq 0\right),
$$

where $N^{\prime}$ is a homogeneous Poisson process with parameter $b-\eta$. Hence, using Minkowski's inequality,

$$
\begin{aligned}
\mathrm{E}\left[\left(\log ^{+} \sup _{t \geq 0}\left(\mathrm{e}^{-\eta t} \tilde{N}_{t}\right)\right)^{2}\right]^{1 / 2} & \leq \log ^{+} M+\mathrm{E}\left[\left(\log ^{+} \sup _{t>0}\left(\frac{N_{0}^{\prime} f(s) \mathrm{d} s}{\int_{0}^{t} f(s) \mathrm{d} s}\right)\right)^{2}\right]^{1 / 2} \\
& =\log ^{+} M+\mathrm{E}\left[\left(\log ^{+} \sup _{t>0}\left(\frac{N_{t}^{\prime}}{t}\right)\right)^{2}\right]^{1 / 2}
\end{aligned}
$$

and the second term on the right-hand side is finite using Lemma 3.2. Hence, $\left(\tilde{N}_{t}, t \geq 0\right)$ satisfies condition (C) since $\mathrm{E}\left[M^{2}\right]<\infty$ and $X^{d}$ as well.

3.3.3. Proof of condition $(C)$ for $X^{g}$. We have

$$
X_{t}^{g}=\sum_{i=1}^{\hat{N}_{t}} \hat{X}_{i}\left(t-\hat{T}_{i}\right)
$$


As in the previous section,

$$
\mathrm{e}^{-\eta t} X_{t}^{g} \leq \mathrm{e}^{-\eta t} \hat{N}_{t}\left(\frac{1}{\hat{N}_{t}} \sum_{i=1}^{\hat{N}_{t}} \hat{Y}_{i}\right) \quad \text { a.s. }
$$

where $\hat{Y}_{i}$ is the total progeny of the conditioned CMJ process $\left(\hat{X}_{i}(t), t \geq 0\right)$. Hence,

$$
\begin{aligned}
\mathrm{E}\left[\left(\log ^{+} \sup _{t \geq 0}\left(\mathrm{e}^{-\eta t} X_{t}^{g}\right)\right)^{2}\right]^{1 / 2} \leq & \mathrm{E}\left[\left(\log ^{+} \sup _{t \geq 0}\left(\mathrm{e}^{-\eta t} \hat{N}_{t}\right)\right)^{2}\right]^{1 / 2} \\
& +\mathrm{E}\left[\left(\log ^{+} \sup _{n>0}\left(\frac{1}{n} \sum_{i=1}^{n} \hat{Y}_{i}\right)\right)^{2}\right]^{1 / 2} .
\end{aligned}
$$

We first prove that the second term on the right-hand side of (3.5) is finite using Lemma 3.1. We only have to check that $\mathrm{E}\left[\hat{Y}_{1}\right]$ is finite. We recall that $\hat{Y}_{1}$ is the total progeny of a splitting tree whose ancestor has random lifespan $R_{1}$ and conditioned on extinction. Conditioning on $R_{1}$, it is also the total progeny of a subcritical Bienaymé-Galton-Watson process starting from a Poisson random variable with mean $R_{1}$. Hence, $\mathrm{E}\left[\hat{Y}_{1}\right]$ is finite if $\mathrm{E}\left[R_{1}\right]$ is finite. As a consequence of (3.2), we have $\mathrm{P}\left(R_{1} \in \mathrm{d} r\right)=\mathrm{e}^{-\eta r} \int_{r}^{\infty} \Lambda(\mathrm{d} z) \mathrm{d} r$ and

$$
\begin{aligned}
\mathrm{E}\left[R_{1}\right] & =\int_{(0, \infty)} \Lambda(\mathrm{d} z) \int_{0}^{z} r \mathrm{e}^{-\eta r} \mathrm{~d} r \\
& =-\int_{(0, \infty)} \Lambda(\mathrm{d} z) \frac{z \mathrm{e}^{-\eta z}}{\eta}+\int_{(0, \infty)} \Lambda(\mathrm{d} z) \frac{1-\mathrm{e}^{-\eta z}}{\eta^{2}} \\
& =\frac{\psi^{\prime}(\eta)-1}{\eta}+\frac{1}{\eta} \\
& =\frac{\psi^{\prime}(\eta)}{\eta} \\
& <\infty
\end{aligned}
$$

We are now interested in the first term on the right-hand side of (3.5). We need to make calculations on $\hat{N}_{t}$, which denotes the total number of times the graftings $T_{i, j}$ are less than or equal to $t$. Recall that, for $i \geq 0$ and $j \geq 1, T_{i, j}=\tau_{i}+\bar{A}_{i, j}$ where $\bar{A}_{i, j}:=A_{i, 1}+\cdots+A_{i, j}$, that $\tau_{i}$ is the birth time of individual $i$, and that $\hat{N}_{t}$ is the sum of the numbers of graftings before $t$ on each of the $X_{t}^{\infty}$ branches. For $i \geq 0$, denote by $\alpha_{1}^{i}, \alpha_{2}^{i}, \ldots$ the birth times of the daughters of individual $i$ and let $\alpha_{0}^{i}=\tau_{i}$. For $k \geq 1$, denote by $\tilde{\tau}_{k}^{i}:=\alpha_{k}^{i}-\alpha_{k-1}^{i}$ the interbirth times. In particular, $\left(\tilde{\tau}_{k}^{i}, i \geq 0, k \geq 1\right)$ are i.i.d. exponential random variables with parameter $\eta$ since we consider an $\eta$-Yule tree.

We enlarge the probability space by redefining the renewal processes $\left(\bar{A}_{i, j}, j \geq 1\right)$ in terms of a doubly indexed sequence of i.i.d. $A$-renewal processes $\left(\mathcal{A}_{i, k}, i \geq 0, k \geq 1\right)$. We define the process $\left(\bar{A}_{i, j}, j \geq 1\right)$ recursively by concatenation of the $\mathcal{A}_{i, k} \mathrm{~s}$ as in Figure 3 . To simplify the notation, we define only $\left(\bar{A}_{0, j}, j \geq 1\right)$, which will be denoted by $\left(\bar{A}_{j}, j \geq 1\right)$. Then,

$$
\bar{A}_{1}^{\prime}:=\inf \left\{t>\alpha_{1} \mid \mathcal{A}_{1} \cap[t,+\infty) \neq \varnothing\right\}, \quad C_{1}:=\# \mathcal{A}_{1} \cap\left[0, \alpha_{1}\right]+1,
$$

and

$$
\bar{A}_{j}:=\inf \left\{t \geq 0 \mid \# \mathcal{A}_{1} \cap[0, t]=j\right\}, \quad j=1, \ldots, C_{1}+1 .
$$




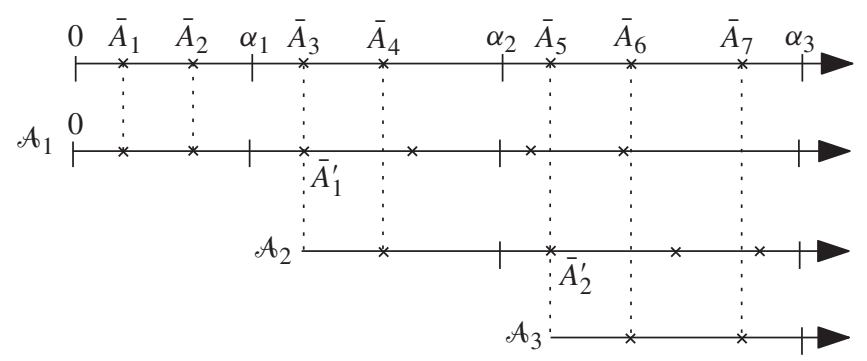

FIGURE 3: Construction of the renewal process $\left(\bar{A}_{j}, j \geq 1\right)$ by concatenation of the renewal processes $\mathcal{A}_{k}$.

Moreover, for $l \geq 1$, if we know $C_{l}$ and $\bar{A}_{l}^{\prime}$, let $r_{l}$ be the unique integer such that $\bar{A}_{l}^{\prime}$ belongs to $\left(\alpha_{r_{l}}, \alpha_{r_{l}+1}\right]$ and define

$$
\begin{aligned}
& \bar{A}_{l+1}^{\prime}:=\bar{A}_{l}^{\prime}+\inf \left\{t>\alpha_{r_{l}+1}-\bar{A}_{l}^{\prime} \mid \mathcal{A}_{r_{l}+1} \cap[t,+\infty) \neq \varnothing\right\}, \\
& C_{l+1}:=\# \mathcal{A}_{r_{l}+1} \cap\left[0, \alpha_{r_{l}+1}-\bar{A}_{l}^{\prime}\right]+1,
\end{aligned}
$$

and

$$
\bar{A}_{C_{1}+\cdots+C_{l}+j}:=\bar{A}_{l}^{\prime}+\inf \left\{t \geq 0 \mid \# \mathcal{A}_{r_{l}+1} \cap[0, t]=j\right\}, \quad j=1, \ldots, C_{l+1} .
$$

Then, $\left(\bar{A}_{j}, j \geq 1\right)$ is an $A$-renewal process because we concatenated the independent renewal processes $\left(\mathcal{A}_{k}\right)$ stopped at the first renewal time after a given time. Indeed, we can see a renewal process as the range of a compound Poisson process whose jumps are distributed as $A_{1}$. The renewal process stopped at the first point after $t$ is then the range of a compound Poisson process stopped at the first hitting time $T$ of $[t, \infty)$, which is a stopping time. Then $\left(\bar{A}_{j}, j \geq 1\right)$, which is the range of the concatenation of independent compound Poisson processes killed at stopping times, is a compound Poisson process, by the strong Markov property. In conclusion, $\left(\bar{A}_{j}, j \geq 1\right)$ is an $A$-renewal process.

According to previous computations, we have

$$
\hat{N}_{t} \leq \sum_{i \geq 0, l \geq 1} C_{l}^{i} \mathbf{1}_{\left\{\alpha_{r_{l, i}}^{i} \leq t\right\}}
$$

where $C_{l}^{i}$ and $r_{l, i}^{i}$ are analogous to the $C_{l}$ and $r_{l}$ notation for individual $i$. Then, if

$$
D_{k}^{i}:=\# \mathcal{A}_{i, k} \cap\left[0, \tilde{\tau}_{k}^{i}\right]+1, \quad k \geq 1, i \geq 0,
$$

since $\alpha_{r_{l, i}+1}^{i}-\bar{A}_{l, i}^{\prime} \leq \alpha_{r_{l, i}+1}^{i}-\alpha_{r_{l, i}}^{i}=\tilde{\tau}_{r_{l, i}}^{i}, C_{l}^{i} \leq D_{r_{l, i}}^{i}$ and

$$
\hat{N}_{t} \leq \sum_{i \geq 0, l \geq 1} D_{r_{l, i}}^{i} \mathbf{1}_{\left\{\alpha_{r_{l, i}}^{i} \leq t\right\}} \leq \sum_{i \geq 0, k \geq 1} D_{k}^{i} \mathbf{1}_{\left\{\alpha_{k}^{i} \leq t\right\}} \quad \text { a.s. }
$$

Moreover, the random variables $\left(D_{k}^{i}, i \geq 0, k \geq 1\right)$ are i.i.d. as 1 plus the value of an $A$-renewal process at an independent exponential time $E$ with parameter $\eta$ :

$$
D:=\sup \left\{j \geq 1, A_{1}+\cdots+A_{j} \leq E\right\}+1 .
$$

The sum on the right-hand side of (3.6) has $2 X_{t}^{\infty}-1$ terms. Indeed, each individual of the Yule tree contributes to $1+n_{i}$ terms in the sum, where $n_{i}$ is the number of daughters of individual $i$ 
born before $t$. Then, there is $X_{t}^{\infty}+\sum_{i=0}^{X_{t}^{\infty}-1} n_{i}$ terms in the sum and $\sum_{i} n_{i}$ is the number of descendants of individual 0 born before $t$, which equals $X_{t}^{\infty}-1$. Hence, using Minkowski's inequality,

$$
\begin{aligned}
\mathrm{E}\left[\left(\log ^{+} \sup _{t \geq 0}\left(\mathrm{e}^{-\eta t} \hat{N}_{t}\right)\right)^{2}\right]^{1 / 2} \leq & \mathrm{E}\left[\left(\log ^{+} \sup _{t \geq 0}\left(\mathrm{e}^{-\eta t}\left(2 X_{t}^{\infty}-1\right)\right)\right)^{2}\right]^{1 / 2} \\
& +\mathrm{E}\left[\left(\log ^{+} \sup _{n>0}\left(\frac{1}{n} \sum_{i=1}^{n} D_{i}\right)\right)^{2}\right]^{1 / 2}
\end{aligned}
$$

where $\left(D_{i}, i \geq 1\right)$ are i.i.d. random variables distributed as $D$. The first term in (3.7) is smaller than $\mathrm{E}\left[\left(\log ^{+}(2 M)\right)^{2}\right]^{1 / 2}$ and the second term is finite by another use of Lemma 3.1 if $\mathrm{E}\left[D_{1}\right]<\infty$. Denote by $\left(C_{t}, t \geq 0\right)$ the renewal process whose arrival times are distributed as $A$. A little calculation from (3.2) gives us that $\mathrm{E}[A]=(m-1) / \eta>0$. Thus, according to Theorem 2.3 of [6, Chapter 5], we have

$$
\lim _{t \rightarrow \infty} \frac{\mathrm{E}\left[C_{t}\right]}{t}=\frac{\eta}{m-1},
$$

and so there exists a $\kappa>0$ such that $\mathrm{E}\left[C_{t}\right] \leq \kappa t$ for $t \geq 0$. Then,

$$
\mathrm{E}\left[D_{1}\right]=1+\mathrm{E}\left[C_{E}\right]=1+\int_{0}^{\infty} \eta \mathrm{e}^{-\eta t} \mathrm{~d} t \mathrm{E}\left[C_{t}\right] \leq 1+\kappa \int_{0}^{\infty} \eta \mathrm{e}^{-\eta t} \mathrm{~d} t=1+\frac{\kappa}{\eta}<\infty .
$$

Finally, the right-hand sides of (3.7) and (3.5) are finite and $X^{g}$ satisfies condition (C).

\section{Proof of Theorem 2.1}

\subsection{Some preliminary lemmas}

We start with some properties about $W$, the scale function associated with $\psi$ and defined by (2.3).

Lemma 4.1. (i) $W(0)=1$,

(ii) $\mathrm{e}^{-\eta t} W(t) \rightarrow c^{-1}$ as $t \rightarrow \infty$,

(iii) $W$ is differentiable and $W \star \Lambda=b W-W^{\prime}$, where ' $\star$ ' is the convolution product.

Proof. (i) We have

$$
\int_{0}^{\infty} \mathrm{e}^{-\lambda t} W(t) \mathrm{d} t=\frac{1}{\psi(\lambda)} \sim \frac{1}{\lambda} \quad \text { as } \lambda \rightarrow \infty
$$

because $\psi(\lambda)=\lambda-b+\int_{(0, \infty)} \mathrm{e}^{-\lambda r} \Lambda(\mathrm{d} r)$. Then, by a Tauberian theorem [2, p. 10], $\lim _{t \rightarrow 0} W(t)=1$.

(ii) (From [18].) For $\lambda>0$, using a Taylor expansion and $\psi(\eta)=0$,

$$
\psi(\lambda+\eta) \sim \lambda \psi^{\prime}(\eta)=\lambda c \quad \text { as } \lambda \rightarrow 0
$$

Then

$$
\int_{0}^{\infty} W(t) \mathrm{e}^{-\eta t} \mathrm{e}^{-\lambda t} \mathrm{~d} t \sim \frac{1}{\lambda c} \quad \text { as } \lambda \rightarrow 0
$$

and another Tauberian theorem entails that $W(t) \mathrm{e}^{-\eta t}$ converges to $1 / c$ as $t \rightarrow \infty$. 
(iii) We first compute the Laplace transform of $W \star \Lambda$. Let $\lambda>\eta$. Then

$$
\begin{aligned}
\int_{0}^{\infty} \mathrm{e}^{-\lambda t} W \star \Lambda(t) \mathrm{d} t & =\int_{0}^{\infty} \mathrm{e}^{-\lambda t} W(t) \mathrm{d} t \int_{(0, \infty)} \mathrm{e}^{-\lambda r} \Lambda(\mathrm{d} r) \\
& =\frac{1}{\psi(\lambda)}(\psi(\lambda)-\lambda+b) .
\end{aligned}
$$

Integrating by parts and using (i) and (ii),

$$
\int_{0}^{\infty} \mathrm{e}^{-\lambda t} W^{\prime}(t) \mathrm{d} t=\left[\mathrm{e}^{-\lambda t} W(t)\right]_{0}^{\infty}+\lambda \int_{0}^{\infty} \mathrm{e}^{-\lambda t} W(t) \mathrm{d} t=-1+\frac{\lambda}{\psi(\lambda)},
$$

and so the Laplace transform of $b W-W^{\prime}$ is $b / \psi(\lambda)+1-\lambda / \psi(\lambda)$, which equals that of $W \star \Lambda$. This completes the proof.

The following lemma deals with the convergence of random series.

Lemma 4.2. Let $\left(\zeta_{i}, i \geq 1\right)$ be a sequence of i.i.d. positive random variables such that $\mathrm{E}\left[\log ^{+} \zeta_{1}\right]$ is finite, and let $\left(\tau_{i}, i \geq 1\right)$ be the arrival times of a Poisson point process with parameter $\rho$ independent of $\left(\zeta_{i}, i \geq 1\right)$. Then, for any $r>0$, the series $\sum_{i \geq 1} \mathrm{e}^{-r \tau_{i}} \zeta_{i}$ converges a.s.

Proof. We have

$$
\sum_{i \geq 1} \mathrm{e}^{-r \tau_{i}} \zeta_{i} \leq \sum_{i \geq 1} \exp \left(-i\left[r \frac{\tau_{i}}{i}-\frac{\log ^{+} \zeta_{i}}{i}\right]\right)
$$

We use the following consequence of Borel-Cantelli's lemma: if $\xi_{1}, \xi_{2}, \ldots$ are i.i.d. nonnegative random variables, $\lim \sup _{n \rightarrow \infty} \xi_{n} / n=0$ or $\infty$ a.s. according to whether $\mathrm{E}\left[\xi_{1}\right]$ is finite or not. We use it with $\xi_{i}=\log ^{+} \zeta_{i}$. Hence, since $\mathrm{E}\left[\log ^{+} \zeta_{1}\right]$ is finite, $\lim _{i \rightarrow \infty} \log ^{+} \zeta_{i} / i=0$ a.s. Moreover, by the strong law of large numbers, $\tau_{i} / i$ converges a.s. to $1 / \rho$ as $i$ goes to $\infty$. Then,

$$
r \frac{\tau_{i}}{i}-\frac{\log ^{+} \zeta_{i}}{i} \rightarrow \frac{r}{\rho}>0 \quad \text { a.s. as } i \rightarrow \infty .
$$

So the series in (4.1) converges a.s.

\subsection{Proof of Theorem 2.1(i)}

In order to find the law of $I(t)$, the total population at time $t$, we use the fact that it is the sum of a Poissonian number of population sizes. More specifically, if we denote by $N_{t}$ the number of populations at time $t,\left(N_{t}, t \geq 0\right)$ is a Poisson process with parameter $\theta$ and, conditionally on $\left\{N_{t}=k\right\}$, the $k$-tuple $\left(T_{1}, \ldots, T_{k}\right)$ has the same distribution as $\left(U_{(1)}, \ldots, U_{(k)}\right)$, which is the reordered $k$-tuple of $k$ independent uniform random variables on $[0, t]$. Hence, conditionally on $\left\{N_{t}=k\right\}$,

$$
I(t) \stackrel{\mathrm{D}}{=} \sum_{i=1}^{k} X_{i}\left(t-U_{(i)}\right) \stackrel{\mathrm{D}}{=} \sum_{i=1}^{k} X_{i}\left(t-U_{i}\right) \stackrel{\mathrm{D}}{=} \sum_{i=1}^{k} X_{i}\left(U_{i}\right),
$$

since all $U_{(i)}$ s appear in the sum and the $U_{i}$ s are independent from the $X_{i}$ s. Hence, conditionally on $\left\{N_{t}=k\right\}, I(t)$ has the same distribution as a sum of $k$ i.i.d. random variables with law $X(U)$. 
Then,

$$
\begin{aligned}
G_{t}(s) & =\sum_{k \geq 0} \mathrm{E}\left[s^{I(t)} \mid N_{t}=k\right] \mathrm{P}\left(N_{t}=k\right) \\
& =\sum_{k \geq 0} \mathrm{E}\left[s^{X_{1}\left(U_{1}\right)}\right]^{k} \frac{(t \theta)^{k}}{k !} \mathrm{e}^{-\theta t} \\
& =\mathrm{e}^{-\theta t} \exp \left(t \theta \mathrm{E}\left[s^{X_{1}\left(U_{1}\right)}\right]\right) .
\end{aligned}
$$

We now compute the law of $X(t)$ for $t>0$ and then we will compute the law of $X_{1}\left(U_{1}\right)$. Using (2.1), (2.2), and Lemma 4.1(iii), we have

$$
\begin{aligned}
\mathrm{P}(X(t)=0) & =\int_{(0, \infty)} \tilde{\mathrm{P}}_{r}(X(t)=0) \frac{\Lambda(\mathrm{d} r)}{b} \\
& =\int_{(0, \infty)} \frac{W(t-r)}{W(t)} \frac{\Lambda(\mathrm{d} r)}{b} \\
& =\frac{1}{b W(t)} W \star \Lambda(t) \\
& =1-\frac{W^{\prime}(t)}{b W(t)}
\end{aligned}
$$

and, for $n \in \mathbb{N}^{*}$,

$$
\begin{aligned}
\mathrm{P}(X(t)=n) & =\int_{(0, \infty)} \tilde{\mathrm{P}}_{r}(X(t)=n) \frac{\Lambda(\mathrm{d} r)}{b} \\
& =\frac{1}{b W(t)}\left(1-\frac{1}{W(t)}\right)^{n-1}\left(b-W(t)^{-1} W \star \Lambda(t)\right) \\
& =\left(1-\frac{1}{W(t)}\right)^{n-1} \frac{W^{\prime}(t)}{b W(t)^{2}} .
\end{aligned}
$$

Now

$$
\mathrm{P}\left(X_{1}\left(U_{1}\right)=0\right)=\frac{1}{t} \int_{0}^{t} \mathrm{P}\left(X_{1}(u)=0\right) \mathrm{d} u=1-\frac{1}{t b} \int_{0}^{t} \frac{W^{\prime}(u)}{W(u)} \mathrm{d} u=1-\frac{\log W(t)}{t b}
$$

because $W(0)=1$. For $n>0$,

$$
\begin{aligned}
\mathrm{P}\left(X_{1}\left(U_{1}\right)=n\right) & =\frac{1}{t} \int_{0}^{t} \mathrm{P}\left(X_{1}(u)=n\right) \mathrm{d} u \\
& =\frac{1}{t} \int_{0}^{t}\left(1-\frac{1}{W(u)}\right)^{n-1} \frac{W^{\prime}(u)}{b W(u)^{2}} \mathrm{~d} u \\
& =\frac{1}{b t} \int_{W(t)^{-1}}^{1} \frac{(1-u)^{n-1}}{n} \mathrm{~d} u \\
& =\frac{(1-1 / W(t))^{n}}{b t n} .
\end{aligned}
$$


We are now able to compute the generating function of $X_{1}\left(U_{1}\right)$. For $s, t>0$,

$$
\begin{aligned}
\mathrm{E}\left[s^{X_{1}\left(U_{1}\right)}\right] & =\frac{1}{b t} \sum_{n \geq 1} \frac{s^{n}}{n}\left(1-\frac{1}{W(t)}\right)^{n}+1-\frac{\log W(t)}{t b} \\
& =1-\frac{1}{b t}\left[\log \left(1-s\left(1-\frac{1}{W(t)}\right)\right)+\log W(t)\right] \\
& =1-\frac{1}{b t} \log (W(t)+s(1-W(t))) .
\end{aligned}
$$

Finally, for $t, s>0$, according to (4.2),

$$
G_{t}(s)=\mathrm{e}^{-\theta t} \exp \left(t \theta \mathrm{E}\left[s^{X_{1}\left(U_{1}\right)}\right]\right)=(W(t)+s(1-W(t)))^{-\theta / b},
$$

which is the probability generating function of a negative binomial distribution with parameters $1-W(t)^{-1}$ and $\theta / b$.

\subsection{Proof of Theorem 2.1(ii)}

We first prove the almost-sure convergence. Splitting $I(t)$ between the surviving and the nonsurviving populations, we have

$$
\mathrm{e}^{-\eta t} I(t)=\sum_{i \geq 1} \mathrm{e}^{-\eta t} Z^{(i)}(t)+\sum_{i \geq 1} \mathrm{e}^{-\eta t} X_{i}\left(t-T_{i}\right) \mathbf{1}_{\left\{t \geq T_{i}\right\} \cap \operatorname{Ext}_{i},},
$$

where, for $i \geq 1$, Ext $_{i}$ denotes the extinction of the process $X_{i}$. We will show that, for each of these two terms, we can exchange the summation and limit, so that, in particular, the second term vanishes as $t \rightarrow \infty$.

We first treat the second term on the right-hand side of (4.3). We have

$$
C_{t}:=\sum_{i \geq 1} X_{i}\left(t-T_{i}\right) \mathbf{1}_{\left\{t \geq T_{i}\right\} \cap \mathrm{Ext}_{i}} \leq \sum_{i \geq 1} \mathbf{1}_{\left\{t \geq T_{i}\right\}} Y_{i} \mathbf{1}_{\mathrm{Ext}_{i}} \quad \text { a.s. }
$$

where $Y_{i}$ is the total progeny of the $i$ th population that does not survive. Moreover, $\mathrm{E}\left[Y_{1} \mathbf{1}_{\mathrm{Ext}}\right] \leq$ $\mathrm{E}\left[Y_{1}\right]$, where $Y_{1}$ is the total progeny of a subcritical Bienaymé-Galton-Watson process so its mean is finite. Hence, since the right-hand side of the previous equation is a compound Poisson process with finite mean, it grows linearly and $\mathrm{e}^{-\eta t} C_{t}$ vanishes as $t \rightarrow \infty$.

To exchange the summation and limit in the first term on the right-hand side of (4.3), we will use the dominated convergence theorem. By Proposition 2.2, we already know that $\mathrm{e}^{-\eta t} Z^{(i)}(t)$ converges a.s. as $t$ goes to $\infty$ to $\mathrm{e}^{-\eta T^{(i)}} E_{i}$. Hence, it is sufficient to prove that

$$
\sum_{i \geq 1} \sup _{t \geq 0}\left(\mathrm{e}^{-\eta t} Z^{(i)}(t)\right)<\infty \quad \text { a.s. }
$$

Since

$$
\sup _{t \geq 0}\left(\mathrm{e}^{-\eta t} Z^{(i)}(t)\right)=\mathrm{e}^{-\eta T^{(i)}} \sup _{t \geq 0}\left(\mathrm{e}^{-\eta t} X_{(i)}(t)\right)
$$

we have

$$
\sum_{i \geq 1} \sup _{t \geq 0}\left(\mathrm{e}^{-\eta t} Z^{(i)}(t)\right)=\sum_{i \geq 1} \mathrm{e}^{-\eta T^{(i)}} J_{i}
$$

where $J_{i}:=\sup _{t \geq 0}\left(\mathrm{e}^{-\eta t} X_{(i)}(t)\right)$ for $i \geq 1$ and $J_{1}, J_{2}, \ldots$ are i.i.d. Thus, using Lemmas 4.2, this series converges a.s. if $\mathrm{E}\left[\log ^{+} J_{1}\right]$ is finite, which is checked thanks to Proposition 2.1(ii). 
Then we get (4.4) and, using the dominated convergence theorem,

$$
I:=\lim _{t \rightarrow \infty} \mathrm{e}^{-\eta t} I(t)=\sum_{i \geq 1} \mathrm{e}^{-\eta T^{(i)}} E_{i} \quad \text { a.s. }
$$

In order to find the law of $I$, we compute its Laplace transform. For $a>0$, using part (i) of this theorem,

$$
\mathrm{E}\left[\mathrm{e}^{-a \mathrm{e}^{-\eta t} I(t)}\right]=G_{t}\left(\mathrm{e}^{-a \mathrm{e}^{-\eta t}}\right)=\left(\mathrm{e}^{-a \mathrm{e}^{-\eta t}}+\left(1-\mathrm{e}^{-a \mathrm{e}^{-\eta t}}\right) W(t)\right)^{-\theta / b}
$$

and

$$
\mathrm{e}^{-a \mathrm{e}^{-\eta t}}+\left(1-\mathrm{e}^{-a \mathrm{e}^{-\eta t}}\right) W(t) \sim 1+a \mathrm{e}^{-\eta t} W(t) \rightarrow 1+\frac{a}{c} \quad \text { as } t \rightarrow \infty
$$

using Lemma 4.1 (ii). Then,

$$
\mathrm{E}\left[\mathrm{e}^{-a I}\right]=\left(\frac{c}{a+c}\right)^{\theta / b},
$$

which is the Laplace transform of a $\operatorname{Gamma}(\theta / b, c)$ random variable.

\section{Other proofs}

\subsection{Proof for Model I}

To prove Theorem 2.2, we will follow Tavaré's proof [22]. We begin with a technical lemma which will be useful in the proof of this theorem and in forthcoming proofs.

Lemma 5.1. Let $\left(T_{i}, i \geq 1\right)$ be the arrival times of a Poisson process with parameter $\rho$, and let $\zeta_{1}, \zeta_{2}, \ldots$ be i.i.d. random variables independent of the $T_{i}$ s. Denote by $g$ the density of $\zeta_{1}$ with respect to the Lebesgue measure and by $F(v):=\mathrm{P}\left(\zeta_{1} \geq v\right)$ its distribution tail. Then, for $r>0,\left(\mathrm{e}^{-r T_{i}} \zeta_{i}, i \geq 1\right)$ are the points of a nonhomogeneous Poisson point process on $(0, \infty)$ with intensity measure $(\rho F(v) / r v) \mathrm{d} v$.

Proof. We first study the collection $\Pi=\left\{\left(T_{i}, \zeta_{i}\right), i \geq 1\right\}$. This is a Poisson point process on $(0, \infty) \times(0, \infty)$ with intensity measure $\rho g(y) \mathrm{d} t \mathrm{~d} y$. Then, $\left(\mathrm{e}^{-r T_{i}} \zeta_{i}, i \geq 1\right)$ is a Poisson point process whose intensity measure is the image of $\rho g(y) \mathrm{d} t \mathrm{~d} y$ by $(t, y) \mapsto \mathrm{e}^{-r t} y$. We now compute it. Let $h$ be a nonnegative mapping. Changing variables, we obtain

$$
\begin{aligned}
\int_{0}^{\infty} \int_{0}^{\infty} h\left(\mathrm{e}^{-r t} y\right) \rho g(y) \mathrm{d} t \mathrm{~d} y & =\frac{\rho}{r} \int_{0}^{\infty} h(v) \mathrm{d} v \int_{0}^{1} g\left(\frac{v}{u}\right) \frac{\mathrm{d} u}{u^{2}} \\
& =\frac{\rho}{r} \int_{0}^{\infty} h(v) \frac{F(v)}{v} \mathrm{~d} v .
\end{aligned}
$$

This completes the proof.

We are now able to prove Theorem 2.2. By Proposition 2.2 and Theorem 2.1,

$$
\begin{aligned}
I(t)^{-1}\left(Z^{(1)}(t), Z^{(2)}(t), \ldots\right) & =\frac{\mathrm{e}^{-\eta t}\left(Z^{(1)}(t), Z^{(2)}(t), \ldots\right)}{\mathrm{e}^{-\eta t} I(t)} \\
& \rightarrow\left(\frac{\sigma_{1}}{\sigma}, \frac{\sigma_{2}}{\sigma}, \ldots\right) \text { a.s. as } t \rightarrow \infty,
\end{aligned}
$$

where $\sigma_{i}:=\exp \left(-\eta T^{(i)}\right) E_{i}$ and $\sigma:=\sum_{i \geq 1} \sigma_{i}$. 
Moreover, the $\left(\sigma_{i}\right)_{i \geq 1}$ are the points of a nonhomogeneous Poisson point process on $(0, \infty)$ with intensity measure $\left(\theta \mathrm{e}^{-c y} / b y\right) \mathrm{d} y$ thanks to Lemma 5.1, with $\rho=\theta \eta / b, r=\eta$, and $F(v)=\mathrm{e}^{-c v}$ because $\left(T^{(i)}\right)_{i \geq 1}$ is a Poisson process of rate $\theta \eta / b$ and $\left(E_{i}\right)_{i \geq 1}$ is an independent sequence of i.i.d. exponential variables with parameter $c$.

According to [3, p. 89], the Poisson point process $\left(\sigma_{i}\right)_{i \geq 1}$ satisfies $\sigma=\sum_{i \geq 1} \sigma_{i}<\infty$ (actually $\sigma$ has a gamma distribution) and the vector

$$
\left(\frac{\sigma_{1}}{\sigma}, \frac{\sigma_{2}}{\sigma}, \ldots\right)
$$

follows the GEM distribution with parameter $\theta / b$ and is independent of $\sigma$.

\subsection{Proof for Model II}

We will prove Theorem 2.3. Recall that in Model II, immigrants are of type $i$ with probability $p_{i}$. Denote by $N^{i}(t)$ the number of immigrants of type $i$ which arrived before time $t$. Then, $\left(N^{i}(t), t \geq 0\right)$ is a Poisson process with parameter $\theta p_{i}$ and the processes $\left(N^{i}, i \geq 1\right)$ are independent. Hence, $I_{1}(t), I_{2}(t), \ldots$ are independent and their asymptotic behaviors are the same as $I(t)$ in Theorem 2.1, replacing $\theta$ with $\theta p_{i}$. Then we have

$$
\mathrm{e}^{-\eta t} I_{i}(t) \rightarrow I_{i} \quad \text { a.s. as } t \rightarrow \infty
$$

for $i \geq 1$, where the $I_{i} \mathrm{~s}$ are independent and $I_{i}$ has a gamma distribution $\Gamma\left(\theta_{i}, c\right)$ (recall that $\left.\theta_{i}=\theta p_{i} / b\right)$.

Moreover,

$$
\mathrm{e}^{-\eta t} I(t) \rightarrow I \quad \text { a.s. as } t \rightarrow \infty
$$

where $I \sim \Gamma(\theta / b, c)$. Therefore, for $r \geq 1$,

$$
\lim _{t \rightarrow \infty} I(t)^{-1}\left(I_{1}(t), \ldots, I_{r}(t)\right)=\left(\frac{I_{1}}{I}, \ldots, \frac{I_{r}}{I}\right) \quad \text { a.s. }
$$

In order to investigate the law of this $r$-tuple, we prove that

$$
I=\sum_{i \geq 1} I_{i} \quad \text { a.s. }
$$

First, by Fatou's lemma,

$$
\liminf _{t \rightarrow \infty} \mathrm{e}^{-\eta t} \sum_{i \geq 1} I_{i}(t) \geq \sum_{i \geq 1} \liminf _{t \rightarrow \infty} \mathrm{e}^{-\eta t} I_{i}(t) \quad \text { a.s. }
$$

and so

$$
I \geq \sum_{i \geq 1} I_{i} \quad \text { a.s. }
$$

Second,

$$
\mathrm{E}\left[\sum_{i \geq 1} I_{i}\right]=\sum_{i \geq 1} \frac{\theta_{i}}{c}=\frac{\theta}{b c}=\mathrm{E}[I] .
$$

The last two equations yield (5.1). For $1 \leq i \leq r$, we can write

$$
\frac{I_{i}}{I}=\frac{I_{i}}{I_{1}+\cdots+I_{r}+I^{*}}
$$


where $I^{*}$ is independent of $\left(I_{i}, 1 \leq i \leq r\right)$ and has a gamma distribution $\Gamma\left(\theta / b-\bar{\theta}_{r}, c\right)$ with $\bar{\theta}_{r}:=\sum_{i=1}^{r} \theta_{i}$. Hence, we can compute the joint density of the $r$-tuple $\left(I_{1} / I, \ldots, I_{r} / I\right)$ as

$$
f\left(x_{1}, \ldots, x_{r}\right)=\frac{\Gamma(\theta / b)}{\Gamma\left(\theta / b-\bar{\theta}_{r}\right) \prod_{i=1}^{r} \Gamma\left(\theta_{i}\right)} x_{1}^{\theta_{1}-1} \cdots x_{r}^{\theta_{r}-1}\left(1-x_{1}-\cdots-x_{r}\right)^{\theta / b-\bar{\theta}_{r}-1}
$$

for $x_{1}, \ldots, x_{r}>0$ satisfying $x_{1}+\cdots+x_{r}<1$. This joint density is exactly that of $\left(P_{1}^{\prime}, \ldots, P_{r}^{\prime}\right)$ defined in the statement of the theorem.

\subsection{Proofs for Model III}

We first prove the almost-sure convergence in Proposition 2.4. In order to do this, we use the same arguments as in the proof of Theorem 2.1(ii): we will use the dominated convergence theorem for the sum

$$
\mathrm{e}^{-\eta t} I(t)=\sum_{i \geq 1} \mathrm{e}^{-\eta t} I_{\Delta_{i}}^{i}\left(t-T_{i}\right) \mathbf{1}_{\left\{t \geq T_{i}\right\}}
$$

As in a previous proof, this sum is bounded by $\sum_{i \geq 1} \mathrm{e}^{-\eta T_{i}} \sup _{t \geq 0}\left(\mathrm{e}^{-\eta t} I_{\Delta_{i}}^{i}(t)\right)$, which, according to Lemma 4.2, is a.s. finite if

$$
\mathrm{E}\left[\log ^{+} \sup _{t \geq 0}\left(\mathrm{e}^{-\eta t} I_{\Delta}^{1}(t)\right)\right]<\infty .
$$

However, $I_{\Delta}^{1}(t)=\sum_{i \geq 1} X^{i}\left(t-\tilde{T}_{i}\right) \mathbf{1}_{\left\{t \geq \tilde{T}_{i}\right\}}$, where, conditionally on $\Delta,\left(\tilde{T}_{i}, i \geq 1\right)$ is a Poisson process with parameter $\Delta$. Hence,

$$
\sup _{t \geq 0}\left(\mathrm{e}^{-\eta t} I_{\Delta}(t)\right) \leq \sum_{i \geq 1} \mathrm{e}^{-\eta \tilde{T}_{i}} \sup _{t \geq 0}\left(\mathrm{e}^{-\eta t} X^{i}(t)\right)=\sum_{i \geq 1} \mathrm{e}^{-\eta \tilde{T}_{i}} J_{i},
$$

where $J_{1}, J_{2}, \ldots$ is an i.i.d. sequence of random variables independent from $\tilde{T}_{1}, \tilde{T}_{2}, \ldots$, distributed as $\sup _{t \geq 0}\left(\mathrm{e}^{-\eta t} X(t)\right)$, where $(X(t), t \geq 0)$ is a homogeneous CMJ process. According to Proposition 2.1(ii), we know that $\mathrm{E}\left[\left(\log ^{+} J_{1}\right)^{2}\right]<\infty$.

We define, for $i \geq 1, \varsigma_{i}:=\mathrm{e}^{-\eta \tilde{T}_{i}} J_{i}$ and $\varsigma:=\sum_{i \geq 1} \varsigma_{i}$, and we have to prove that $\mathrm{E}\left[\log ^{+} \varsigma\right]$ is finite. To do this, we first work conditionally on $\Delta$. According to Lemma 5.1, we know that $\left(\varsigma_{i}, i \geq 1\right)$ are the points of a nonhomogeneous Poisson process on $(0, \infty)$ with intensity measure $(\Delta L(v) / \eta v) \mathrm{d} v$, where $L(v):=\mathrm{P}(J \geq v)$. Then, using the inequality

$$
\log ^{+}(x+y) \leq \log ^{+} x+\log ^{+} y+\log 2, \quad x, y \geq 0,
$$

we have

$$
\mathrm{E}\left[\log ^{+} \varsigma\right] \leq \log 2+\mathrm{E}\left[\log ^{+} \sum_{i \geq 1} \varsigma_{i} \mathbf{1}_{\left\{\varsigma_{i} \leq 1\right\}}\right]+\mathrm{E}\left[\log ^{+} \sum_{i \geq 1} \varsigma_{i} \mathbf{1}_{\left\{\varsigma_{i}>1\right\}}\right] .
$$

We first consider the second term on the right-hand side:

$$
\mathrm{E}\left[\log ^{+} \sum_{i \geq 1} \varsigma_{i} \mathbf{1}_{\left\{\varsigma_{i} \leq 1\right\}}\right] \leq \mathrm{E}\left[\sum_{i \geq 1} \varsigma_{i} \mathbf{1}_{\left\{\varsigma_{i} \leq 1\right\}}\right]=\int_{0}^{1} v \frac{\Delta}{\eta} \frac{L(v)}{v} \mathrm{~d} v \leq \frac{\Delta}{\eta} .
$$

Then, we compute the third term on the right-hand side of (5.2): if $A:=\sup _{i} \varsigma_{i}$,

$$
\begin{aligned}
\mathrm{E}\left[\log ^{+} \sum_{i \geq 1} \varsigma_{i} \mathbf{1}_{\left\{\varsigma_{i}>1\right\}}\right] & \leq \mathrm{E}\left[\log ^{+}\left(A \#\left\{i \geq 1 \mid \varsigma_{i}>1\right\}\right)\right] \\
& \leq \mathrm{E}\left[\log ^{+} A\right]+\mathrm{E}\left[\log ^{+} \#\left\{i \geq 1 \mid \varsigma_{i}>1\right\}\right] .
\end{aligned}
$$


Furthermore, the number of $\varsigma_{i}$ greater than 1 has a Poisson distribution with parameter

$$
\int_{1}^{\infty} \frac{\Delta L(v)}{\eta v} \mathrm{~d} v
$$

Since $\mathrm{E}\left[\log ^{+} J\right]<\infty$,

$$
\int_{0}^{\infty} \mathrm{P}\left(\log ^{+} J \geq s\right) \mathrm{d} s=\int_{0}^{\infty} \mathrm{P}\left(J \geq \mathrm{e}^{s}\right) \mathrm{d} s=\int_{1}^{\infty} \frac{L(v)}{v} \mathrm{~d} v<\infty .
$$

Then,

$$
\mathrm{E}\left[\log ^{+} \#\left\{i \geq 1 \mid \varsigma_{i}>1\right\}\right] \leq \mathrm{E}\left[\#\left\{i \geq 1 \mid \varsigma_{i}>1\right\}\right]=\frac{\Delta}{\eta} \int_{1}^{\infty} \frac{L(v)}{v} \mathrm{~d} v \leq C \Delta,
$$

where $C$ is a finite constant which does not depend on $\Delta$.

We now want to study $A$, i.e.

$$
\mathrm{P}(A \leq x)=\mathrm{P}\left(\#\left\{i \geq 1 \mid \varsigma_{i}>x\right\}=0\right)=\exp \left(-\int_{x}^{\infty} \frac{\Delta}{\eta} \frac{L(v)}{v} \mathrm{~d} v\right), \quad x>0,
$$

so that

$$
\mathrm{P}(A \in \mathrm{d} x)=\frac{\Delta}{\eta} \frac{L(x)}{x} \exp \left(-\int_{x}^{\infty} \frac{\Delta}{\eta} \frac{L(v)}{v} \mathrm{~d} v\right) \mathrm{d} x
$$

Then,

$$
\begin{aligned}
\mathrm{E}\left[\log ^{+} A\right] & =\int_{1}^{\infty} \log x \frac{\Delta}{\eta} \frac{L(x)}{x} \exp \left(-\int_{x}^{\infty} \frac{\Delta}{\eta} \frac{L(v)}{v} \mathrm{~d} v\right) \mathrm{d} x \\
& \leq \frac{\Delta}{\eta} \int_{1}^{\infty} \log x \frac{L(x)}{x} \mathrm{~d} x \\
& =\frac{\Delta}{\eta} \int_{0}^{\infty} u L\left(\mathrm{e}^{u}\right) \mathrm{d} u \\
& \leq \frac{\Delta}{\eta} \int_{0}^{\infty} u \mathrm{P}\left(\log ^{+} J \geq u\right) \mathrm{d} u \\
& \leq C^{\prime} \Delta,
\end{aligned}
$$

where $C^{\prime}$ is a finite constant since $\mathrm{E}\left[\left(\log ^{+} J\right)^{2}\right]<\infty$ according to Proposition 2.1(ii). Hence, with (5.3), (5.4), and (5.5), we have

$$
\mathrm{E}\left[\log ^{+} \varsigma \mid \Delta\right] \leq \log 2+C^{\prime \prime} \Delta .
$$

Then, $\mathrm{E}\left[\log ^{+} \varsigma\right]$ is finite because $\mathrm{E}[\Delta]=\theta^{-1} \int_{0}^{\infty} x^{2} f(x) \mathrm{d} x<\infty$, and, using the dominated convergence theorem, $\mathrm{e}^{-\eta t} I(t)$ converges a.s. toward $\sigma=\sum_{i \geq 1} \mathrm{e}^{-\eta T_{i}} G_{i}$ as $t \rightarrow \infty$.

We now compute the law of the limit $\sigma$. We define $\sigma_{i}:=\exp \left(-\eta T_{i}\right) G_{i}$ for $i \geq 1$. Then, using Lemma 5.1, $\left(\sigma_{i}\right)_{i \geq 1}$ are the points of a nonhomogeneous Poisson point process on $(0, \infty)$ with intensity measure $(\theta F(y) / \eta y) \mathrm{d} y$, where $F(y)=\mathrm{P}(G \geq y)$. To compute the Laplace transform of $\sigma$, we use the exponential formula for Poisson processes: for $s>0$,

$$
\mathrm{E}\left[\mathrm{e}^{-s \sigma}\right]=\exp \left(-\frac{\theta}{\eta} \int_{0}^{\infty} \frac{F(v)}{v}\left(1-\mathrm{e}^{-s v}\right) \mathrm{d} v\right) .
$$


To obtain the expectation of $\sigma$, we differentiate the last displayed equation at 0 :

$$
\mathrm{E}[\sigma]=\frac{\theta}{\eta} \int_{0}^{\infty} F(v) \mathrm{d} v=\frac{\theta}{\eta} \mathrm{E}[G]
$$

and

$$
\mathrm{E}[G]=\theta^{-1} \int_{0}^{\infty} x f(x) \frac{x}{b} \frac{1}{c} \mathrm{~d} x<\infty .
$$

Hence,

$$
\mathrm{E}[\sigma]=\frac{1}{\eta b c} \int_{0}^{\infty} x^{2} f(x) \mathrm{d} x<\infty,
$$

which completes the proof of Proposition 2.4.

It remains to prove Theorem 2.4 , that is, to show that the vector $\left(Z^{1}(t), Z^{2}(t), \ldots\right) / I(t)$ converges a.s. to a Poisson point process with intensity measure $(\theta F(y) / \eta y) \mathrm{d} y$. It is straightforward using previous calculations, Propositions 2.3 and 2.4.

Remark 5.1. Thanks to similar calculations as in Theorem 2.1(i), we can compute the generating function of $I(t)$, i.e.

$$
\mathrm{E}\left[s^{I(t)}\right]=\exp \left(-\int_{0}^{t} \mathrm{~d} u\left(\theta-\int_{0}^{\infty} \frac{x f(x)}{(W(u)(1-s)+s)^{x / b}} \mathrm{~d} x\right)\right), \quad s \in[0,1],
$$

and we can deduce the law of $\sigma$ in another way.

\section{Acknowledgements}

I would like to thank my supervisor, Amaury Lambert, for his very helpful remarks. I would also like to thank the anonymous referee for his/her careful reading and advice.

\section{References}

[1] Athreya, K. B. And Ney, P. E. (1972). Branching Processes. Springer, New York.

[2] Bertoin, J. (1996). Lévy Processes (Camb. Tracts Math. 121). Cambridge University Press.

[3] Bertoin, J. (2006). Random Fragmentation and Coagulation Processes (Camb. Stud. Adv. Math. 102). Cambridge University Press.

[4] Caswell, H. (1976). Community structure: a neutral model analysis. Ecological Monographs 46, 327-354.

[5] Donnelly, P. and Tavaré, S. (1986). The ages of alleles and a coalescent. Adv. Appl. Prob. 18, 1-19. (Correction: 18 (1986), 1023.)

[6] Durrett, R. (1999). Essentials of Stochastic Processes. Springer, New York.

[7] Ethier, S. N. (1990). The distribution of the frequencies of age-ordered alleles in a diffusion model. Adv. Appl. Prob. 22, 519-532.

[8] Fisher, R. A., Corbet, A. S. And Williams, C. B. (1943). The relation between the number of species and the number of individuals in a random sample of an animal population. J. Animal Ecology 12, 42-58.

[9] Geiger, J. And Kersting, G. (1997). Depth-first search of random trees, and Poisson point processes. In Classical and Modern Branching Processes (Minneapolis, MN, 1994; IMA Vol. Math. Appl. 84), Springer, New York, pp. 111-126.

[10] Harris, T. E. (1963). The Theory of Branching Processes. Springer, Berlin.

[11] HubBell, S. (2001). The Unified Neutral Theory of Biodiversity and Biogeography. Princeton University Press, NJ.

[12] Jagers, P. (1975). Branching Processes with Biological Applications. Wiley-Interscience, London.

[13] Kallenberg, O. (2002). Foundations of Modern Probability, 2nd edn. Springer, New York.

[14] Karlin, S. And McGregor, J. (1967). The number of mutant forms maintained in a population. In Proc. 5th Berkeley Symp. Math. Statist. Prob., Vol. IV, University of California Press, Berkeley, CA, pp. 415-438.

[15] Kendall, D. G. (1948). On some modes of population growth leading to R. A. Fisher's logarithmic series distribution. Biometrika 35, 6-15. 
[16] Lambert, A. (2008). Population dynamics and random genealogies. Stoch. Models 24, 45-163.

[17] LAMBERT, A. (2010). Species abundance distributions in neutral models with immigration or mutation and general lifetimes. Preprint. Available at http://www.proba.jussieu.fr/pageperso/amaury/index_fichiers/MigraMut.pdf. To appear in J. Math. Biol.

[18] Lambert, A. (2010). The contour of splitting trees is a Lévy process. Ann. Prob. 38, 348-395.

[19] MacArthur, R. H. And Wilson, E. O. (1967). The Theory of Island Biogeography. Princeton University Press, NJ.

[20] Nerman, O. (1981). On the convergence of supercritical general (C-M-J) branching processes. Z. Wahrscheinlichkeithsth. 57, 365-395.

[21] Revuz, D. ANd Yor, M. (1999). Continuous Martingales and Brownian Motion, 3rd edn. Springer, Berlin.

[22] TAvaré, S. (1987). The birth process with immigration, and the genealogical structure of large populations. J. Math. Biol. 25, 161-168.

[23] Tavaré, S. (1989). The genealogy of the birth, death, and immigration process. In Mathematical Evolutionary Theory, Princeton University Press, Princeton, NJ, pp. 41-56.

[24] Volkov, I., Banavar, J. R., Hubbell, S. P. and Maritan, A. (2003). Neutral theory and relative species abundance in ecology. Nature 424, 1035-1037.

[25] Watterson, G. A. (1974). Models for the logarithmic species abundance distributions. Theoret. Pop. Biol. 6, 217-250.

[26] Watterson, G. A. (1974). The sampling theory of selectively neutral alleles. Adv. Appl. Prob. 6, $463-488$. 